Research Article

\title{
Optimal Investment of DC Pension Plan under Incentive Schemes and Loss Aversion
}

\author{
Yinghui Dong (D, Wenxin Lv, Siyuan Wei, and Yeyang Gong \\ Department of Mathematics and Physics, Suzhou University of Science and Technology, Suzhou 215009, China \\ Correspondence should be addressed to Yinghui Dong; dongyinghui1030@163.com
}

Received 17 April 2020; Accepted 4 May 2020; Published 18 May 2020

Guest Editor: Wenguang Yu

Copyright (c) 2020 Yinghui Dong et al. This is an open access article distributed under the Creative Commons Attribution License, which permits unrestricted use, distribution, and reproduction in any medium, provided the original work is properly cited.

\begin{abstract}
We investigate the DC pension manager's portfolio problem when the manager is remunerated through two schemes for DC pension managerial compensation under loss aversion and minimum guarantee. We apply the concavification technique and a static Lagrangian technique to solve the problem and derive the closed-form representation of the optimal wealth and portfolio processes. Theoretical and numerical results show that the incentive schemes can significantly impact the distribution of the optimal terminal wealth.
\end{abstract}

\section{Introduction}

As special financial institutions, risk management of insurance companies is the important guarantee of business security. In the existing references, some focus on the pricing aspect, see Dong [1], Yu et al. [2], and Zhang et al. [3], while others focus on the optimal problems, see Dong et al. [4], Dong and Zheng [5, 6], Sun et al. [7], and Yu et al. [8]. The objective of the present paper is to develop optimal asset management strategies for the DC pension plan.

Due to declining mortality and low interest rates, the literature on the asset allocation of defined contribution (DC) pension plans has grown up significantly in recent years. In a DC plan, the member contributes part of the salary to the pension account and upon retirement, the member can receive regular income from the pension account. The problem of optimal allocation during the accumulation phase for the DC pension plan has attracted a lot of attention, see Boulier et al. [9], Chen et al. [10], Zeng et al. [11], and Wang et al. [12].

The impact of the performance of a fund with respect to a benchmark on the asset management has been extensively investigated recently. The managers are often paid by an incentive scheme that depends on the performance of the fund they manage in order to inspire them to greater efforts. Such a scheme is made up of two components: a management fee, which is proportional to the fund wealth, and an incentive fee which is composed of a combination of some options on the fund, see, for example, Basek et al. [13, 14], Carpenter [15], Basak et al. [16], and Chen and Pennacchi [17]. Incentive schemes of the manager significantly affect the investment strategy. It has been believed that the asset manager may be motivated to take risk in excess by incentive schemes. However, little focuses on the performance fee schemes in DC pension plan management, since people argue that the performance fee may trigger managers to take excessive risk, which may lead to heavy losses in DC pension plans. A typical fee structure of defined contribution (DC) pension plan is an asset-based single management fee. However, managers typically perform poorly and cannot keep up with benchmarks under a scheme with lower incentives. It is widely believed that a performance-based and option-like fee may create greater incentives for a manager to exert more efforts in terms of investment.

As pointed out by $\mathrm{He}$ et al. [18], in the late 1990s, a performance-based fee structure was approved as a managerial incentive in DC pension plan management. However, the performance fee could induce managers to gamble at the expense of DC pension members. The purpose of a DC pension plan is to provide an adequate income for a DC member after retirement. Hence, to ensure safety of a DC 
pension, the performance fee scheme has not been implemented in most DC pension funds. He et al. [18] consider the optimal allocation of the DC pension fund for a manager under two fee structures: the single management fee and the mixed scheme of both management and performance fees. They compare the utilities of both fund managers and members under the two schemes and they state the win-win situation of implementing performance-based incentives in DC pension plan management. Furthermore, He et al. [18] consider the PI constraint, which can well protect the members' benefits by keeping the optimal terminal wealth always above the minimum guarantee, see Basek and Shapiro [13].

Prospect theory (PT), proposed by Kahneman and Tversky [19] and by Tversky and Kahneman [20], is one of the most notable theories in behavioral economics. With the framework of PT, investors base decisions on comparisons with a certain predefined reference point (also known as benchmark) rather than evaluating absolute level of total wealth itself. PT also suggests that individuals tend to be loss averse, that is, to be more sensitive to a loss than to a gain of the same amount. This loss aversion and riskseeking behaviour is described in [20] by an asymmetric S-shaped utility function, convex in the domain of losses and concave in the domain of gains. In this paper, we extend the findings by $\mathrm{He}$ et al. [18] by incorporating loss aversion into modelling, which makes our optimization problem much more complex than that of [18]. We employ the martingale approach and the concavification technique to derive the optimal wealth and portfolio processes under a PI constraint and loss aversion. Although Dong and Zheng [5, 6] and Guan and Liang [21] also investigated the optimal allocation of the DC pension plan under loss aversion, they do not consider the remuneration schemes of DC pension.

The main contribution of this paper is that we have solved an optimization problem of a DC plan under two different kinds of remuneration schemes with the framework of PT. The rest of the paper is organized as follows. In Section 2, we formulate a DC investment problem under loss aversion and two different types of remuneration schemes. In Section 3, we apply the concavification technique and the martingale method to solve the optimization problem and derive closed-form expressions for the optimal wealth process and optimal control. In Section 4, we perform some numerical analysis and discuss the impacts of loss aversion and remuneration schemes on the distribution of the optimal terminal wealth. Section 5 concludes the paper.

\section{The Model}

Let $(\Omega, \mathscr{F}, \mathbb{F}, P)$ be a filtered complete probability space with the filtration $\mathbb{F}:=\left\{\mathscr{F}_{t} \mid 0 \leq t \leq T\right\}$ being the natural filtration generated by a standard Brownian motion $\{W(t)\}_{0 \leq t \leq T}$ and satisfying the usual conditions. Assume that all random variables and stochastic processes in this paper are well defined in this probability space. The DC pension fund starts at time 0 , and the retirement time is $T$.
Let the financial market consist of one riskless savings account $S_{0}(t)$ and one risky asset $S_{1}(t)$.

The price of the risk-free asset $\left\{S_{0}(t)\right\}_{t \geq 0}$ satisfies the following differential equation:

$$
\mathrm{d} S_{0}(t)=r S_{0}(t) \mathrm{d} t
$$

where $r$ is a riskless interest rate. The price of the risky asset $\left\{S_{1}(t)\right\}_{t \geq 0}$ is modelled by the following stochastic differential equation (SDE):

$$
\begin{aligned}
\mathrm{d} S_{1}(t) & =S_{1}(t)(\mu \mathrm{d} t+\sigma \mathrm{d} W(t)) \\
& =S_{1}(t)(r \mathrm{~d} t+\sigma(\mathrm{d} W(t)+\vartheta \mathrm{d} t)),
\end{aligned}
$$

where $\mu>r$ is the expected return of the risky asset, $\sigma>0$ is the volatility of the risky asset, and $\vartheta=(\mu-r)^{\sigma}$ is the market price of risk.

In a DC plan, the pension members contribute continuously to the pension plan before retirement time $T$. Let $c(t)>0$ denote the aggregated amount of money contributed at time $t$ of a cohort of fund participants. As explained in [6], individual members' contribution rates may be random over the time, but the accumulated cash income of a large pension fund is in general deterministic and stable. We therefore, for simplicity, assume $c(t) \equiv c$ is a constant. Assume that there are no transaction costs or taxes in the financial market. The pension account is endowed with an initial endowment $x_{0} \geq 0$.

Let $\pi(t)$ be the total amount allocated to the risky asset at time $t \in[0, T]$. Then, the wealth process $\left\{X^{\pi}(t)\right\}_{0 \leq t \leq T}$ with the initial value $X^{\pi}(0)=0$ satisfies the following controlled SDE:

$$
d X^{\pi}(t)=r X^{\pi}(t) d t+\pi(t) \sigma(d W(t)+\vartheta d t)+c d t .
$$

Let $H(t)$ be the discounted value at time $t$ of total pension contribution from $t$ to $T$, where

$$
H(t)=c \int_{t}^{T} e^{-r(s-t)} \mathrm{d} s=\frac{c}{r}\left(1-e^{-r(T-t)}\right) .
$$

Traditionally, the fund manager charges a management fee as a reward, which is a fixed proportion of terminal fund wealth. More specifically, under this single management fee scheme, the payoff to the DC pension manager is

$$
\Psi_{1}\left(X^{\pi}(T)\right)=k_{1} X^{\pi}(T),
$$

where $k_{1}>0$ is the rate of the management fee.

It is well known that the performance fee may lead to a greater incentive for the fund manager. Following [18], we consider an option-like incentive scheme (also called mixed scheme) and let $M(T)$ be the benchmark for the availability of the performance fee. Assume that $M(T)$ is the expected accumulation of contributions until time $T$ under the reasonable accumulation rate:

$$
M:=M(T)=x_{0} e^{\bar{r} T}+\frac{c}{\bar{r}}\left(e^{\bar{r} T}-1\right)
$$

where $\bar{r}$ is the accumulation rate. Under the mixed scheme, the payoff to the DC pension manager is 


$$
\Psi_{2}\left(X^{\pi}(T)\right)= \begin{cases}k_{2} X^{\pi}(T), & X^{\pi}(T)<M, \\ k_{2} X^{\pi}(T)+k_{3}\left(X^{\pi}(T)-M\right), & X^{\pi}(T) \geq M,\end{cases}
$$

where $0<k_{2} \leq k_{1}$ is the rate of the management fee under the mixed scheme and $k_{3}>0$ is the rate of the performance fee. It is obvious that the manager receives a fixed proportion of terminal wealth and a variable component only in case the DC pension fund outperforms the benchmark $M$ at time $T$.

The DC pension fund is supposed to meet DC members' elementary needs. Therefore, a downside protection $\bar{M}$ should be included in DC pension fund management. We next define the set of admissible trading strategies.

Definition 1. A portfolio strategy $\{\pi=\pi(t): t \in[0, T]\}$ is said to be admissible if, for all $t \in[0, T], \pi(t) \in \mathscr{F}_{t}$, with $\int_{0}^{T} \pi^{2}(t) \mathrm{d} t<+\infty, \quad$ a.s., and $X^{\pi}(T)$ satisfies (3), $X^{\pi}(T) \geq \bar{M}$. We denote the set of admissible portfolio strategies by $\mathscr{A}$.

Consider a utility function defined by

$$
U(x)= \begin{cases}-B(\theta-x)^{\gamma_{1}}, & 0 \leq x<\theta, \\ (x-\theta)^{\gamma_{2}}, & x \geq \theta,\end{cases}
$$

where $\theta>0$ is a reference point, $B>1$ is called the loss aversion degree, $0<\gamma_{1}<1,0<\gamma_{2}<1$ are the constants, $\gamma_{1}$ characterizes the degree of loss aversion, and $\gamma_{2}$ characterizes the degree of risk aversion, respectively.

For each $i=1,2$, we consider the following optimization problem with minimum guarantee:

$$
\begin{cases}\max _{\pi \in \mathscr{A}} & E\left[U\left(\Psi_{i}\left(X^{\pi}(T)\right)\right)\right] \\ \text { s.t. } & X^{\pi}(T) \text { satisfies (3) with } X^{\pi}(T) \geq \bar{M}, \text { a.s } \ldots\end{cases}
$$

Since the market is complete, the unique pricing kernel is as follows:

$$
\begin{aligned}
& \xi(t)=e^{-\left(r+\left(\vartheta^{2} / 2\right)\right) t-\vartheta W(t)}, \\
& \xi(0)=1,0 \leq t \leq T
\end{aligned}
$$

where $\xi(t)$ is the Arrow-Debreu value per probability unit of a security which pays out 1 at time $t$ if the scenario $\omega$ happens, and 0 else. As this value is high in a recession and low in prosperous times, $\xi(t)$ directly reflects the overall state of the economy.

Note that the shapes of $U\left(\Psi_{1}(\cdot)\right)$ and $U\left(\Psi_{2}(\cdot)\right)$ depend on the relationship of the parameters $k_{1}, k_{2}, M$, and $\bar{M}$. For simplicity, here we assume that $k_{1} \bar{M} \leq \theta \leq k_{2} M$. For other cases, the analysis is similar.

Let

$$
\begin{aligned}
& G_{1}(x) \doteq U\left(\Psi_{1}(x)\right)= \begin{cases}-B\left(\theta-k_{1} x\right)^{\gamma_{1},} & 0 \leq x<\frac{\theta}{k_{1},} \\
\left(k_{1} x-\theta\right)^{\gamma_{2},} & x \geq \frac{\theta}{k_{1},}\end{cases} \\
& G_{2}(x) \doteq U\left(\Psi_{2}(x)\right)= \begin{cases}-B\left(\theta-k_{2} x\right)^{\gamma_{1},} & 0 \leq x<\frac{\theta}{k_{2},} \\
\left(k_{2} x-\theta\right)^{\gamma_{2},} & \frac{\theta}{k_{2}} \leq x<M, \\
\left(k_{2} x+k_{3}(x-M)-\theta\right)^{\gamma_{2},} & x \geq M .\end{cases}
\end{aligned}
$$

In the complete market, problem (9) can be rewritten as follows:

$$
\begin{cases}\max _{X^{\pi}(T)} & E\left[G_{i}\left(X^{\pi}(T)\right)\right] \\ \text { s.t. } & E\left[\xi(T) X^{\pi}(T)\right] \leq x_{0}, X^{\pi}(T) \geq \bar{M}, \text { a.s... }\end{cases}
$$

\section{Optimal Trading Strategy under Loss Aversion}

In this section, we will solve the optimization problem (9) and derive the optimal wealth and portfolio processes.

Denote by

$$
k=\frac{G_{2}(M)-G_{2}(\bar{M})}{M-\bar{M}}
$$

the slope of the straight line linking points $\left(\bar{M}, G_{2}(\bar{M})\right)$ and $\left(M, G_{2}(M)\right)$.

\section{Lemma 1}

(1) For $k_{1} \bar{M} \leq \theta$, there exists a unique solution $\left(\theta / k_{1}\right)<z<\infty$ to the equation:

$$
G_{1}(x)-(x-\bar{M}) G_{1}^{\prime}(x)-G_{1}(\bar{M})=0 .
$$

(2) Let $k$ be defined by (13). For $k \geq G_{2}^{\prime}(M-)$, there exists a unique $\left(\theta / k_{2}\right)<\bar{z} \leq M$ satisfying

$$
G_{2}(\bar{z})-G_{2}(\bar{M})-G_{2}^{\prime}(\bar{z})(\bar{z}-\bar{M})=0 .
$$

(3) For $k \leq G_{2}^{\prime}(M+)$, there exists a unique $\tilde{z} \geq M$ satisfying

$$
G_{2}(\tilde{z})-G_{2}(\bar{M})-G_{2}^{\prime}(\tilde{z})(\widetilde{z}-\bar{M})=0 .
$$

Proof. We first prove the first part. Denote $f(x)=G_{1}(x)-(x-\bar{M}) G_{1}^{\prime}(x)-G_{1}(\bar{M})$. It is easy to check that $f(x)$ is a decreasing, continuous function in $\left(\left(\theta / k_{1}\right), \infty\right)$ 
and $f\left(\theta / k_{1}\right)<0, \lim _{x \longrightarrow \infty} f(x)=\infty$. Therefore, there exists unique $\left(\theta / k_{1}\right)<z<\infty$ such that $f(z)=0$ (see Figure 1), which concludes the proof of the first part. Similarly, we can prove the second and the third parts (see Figures 2 and 3). Here, we omit the details.

Lemma 2. For $G_{2}^{\prime}(M-)<G_{2}^{\prime}(M+)$, there exists a unique pair $\left(z_{1}, z_{2}\right)$ with $\left(\theta / k_{2}\right)<z_{1}<M<z_{2}$ satisfying

$$
\frac{G_{2}\left(z_{2}\right)-G_{2}\left(z_{1}\right)}{z_{2}-z_{1}}=G_{2}^{\prime}\left(z_{1}\right)=G_{2}^{\prime}\left(z_{2}\right) \text {. }
$$

Proof. Note that $G_{2}^{\prime}(x)$ is continuous and strictly decreasing on $\left(\left(k_{2} / \theta\right), M\right)$ and $(M, \infty)$, respectively. Furthermore,

$$
G_{2}^{\prime}(M-)<G_{2}^{\prime}(M+), \lim _{x \longrightarrow\left(\theta / k_{2}\right)^{+}} G_{2}^{\prime}(x)=+\infty, \lim _{x \longrightarrow+\infty} G_{2}^{\prime}(x)=0 .
$$

Therefore, $G_{2}^{\prime}(x)$ on $\left(\left(k_{2} / \theta\right), M\right)$ and $G_{2}^{\prime}(x)$ on $(M, \infty)$ have strictly decreasing inverse functions and there must exist unique $\left(\theta / k_{2}\right)<\eta_{1}<M$ and $\eta_{2}>M$, such that

$$
\begin{aligned}
& G_{2}^{\prime}\left(\eta_{1}\right)=G_{2}^{\prime}(M+), \\
& G_{2}^{\prime}\left(\eta_{2}\right)=G_{2}^{\prime}(M-) .
\end{aligned}
$$

For any $y \in\left[G_{2}^{\prime}(M-), G_{2}^{\prime}(M+)\right]$, we define $\left(\theta / k_{2}\right)<$ $c_{1}(y) \leq M \leq c_{2}(y)$ as

$$
G_{2}^{\prime}\left(c_{1}(y)\right)=G_{2}^{\prime}\left(c_{2}(y)\right)=y .
$$

It is obvious that $c_{1}(y)$ and $c_{2}(y)$ are strictly decreasing and continuous on $\left[G_{2}^{\prime}(M-), G_{2}^{\prime}(M+)\right]$ and

$$
\begin{aligned}
y \rightarrow G_{2}^{\prime}(M-) & c_{1}(y)=M, \\
& \lim _{y \rightarrow G_{2}^{\prime}(M-)} c_{2}(y)=\eta_{2}, \\
& \lim _{y \rightarrow G_{2}^{\prime}(M+)} c_{1}(y)=\eta_{1}, \\
& \lim _{y \rightarrow G_{2}^{\prime}(M+)} c_{2}(y)=M .
\end{aligned}
$$

Define

$$
\mathscr{K}(y)=\frac{G_{2}\left(c_{2}(y)\right)-G_{2}\left(c_{1}(y)\right)}{c_{2}(y)-c_{1}(y)} .
$$

It is easy to check that $\mathscr{K}(y)$ is continuous on $\left[G_{2}^{\prime}(M-), G_{2}^{\prime}(M+)\right]$. Using the concave inequalities, we have

$$
\begin{aligned}
G_{2}^{\prime}(M+)\left(c_{2}(y)-M\right) & >G_{2}\left(c_{2}(y)\right)-G_{2}(M) \\
& >G_{2}^{\prime}\left(c_{2}(y)\right)\left(c_{2}(y)-M\right) \\
& \geq G_{2}^{\prime}(M-)\left(c_{2}(y)-M\right), \\
G_{2}^{\prime}(M+)\left(M-c_{1}(y)\right) & \geq G_{2}^{\prime}\left(c_{1}(y)\right)\left(M-c_{1}(y)\right) \\
& >G_{2}(M)-G_{2}\left(c_{1}(y)\right) \\
& >G_{2}^{\prime}(M-)\left(M-c_{1}(y)\right) .
\end{aligned}
$$

It follows from the above two inequalities that

$$
G^{\prime}(M+)>\mathscr{K}(y)>G^{\prime}(M-) .
$$

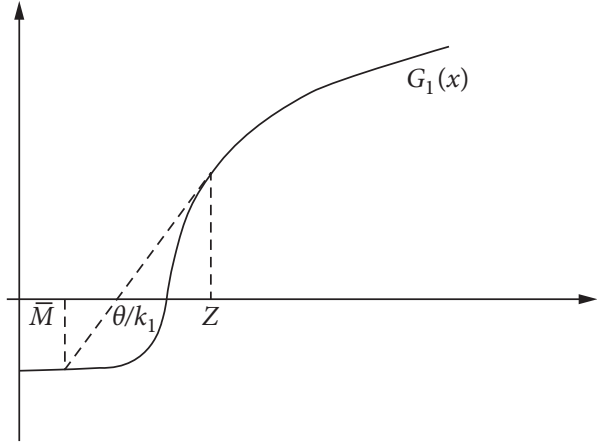

Figure 1: The root to equation (14).

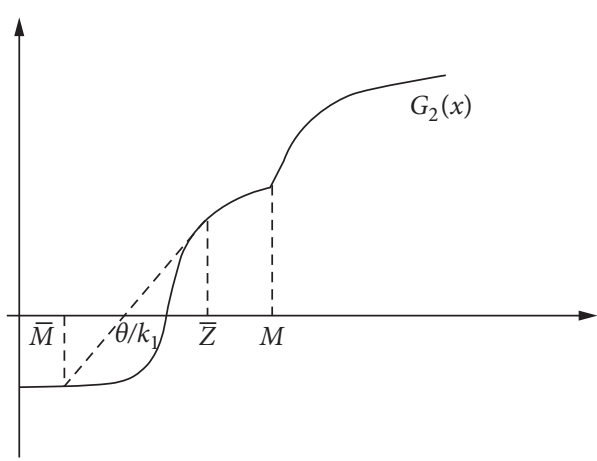

Figure 2: The root to equation (15).

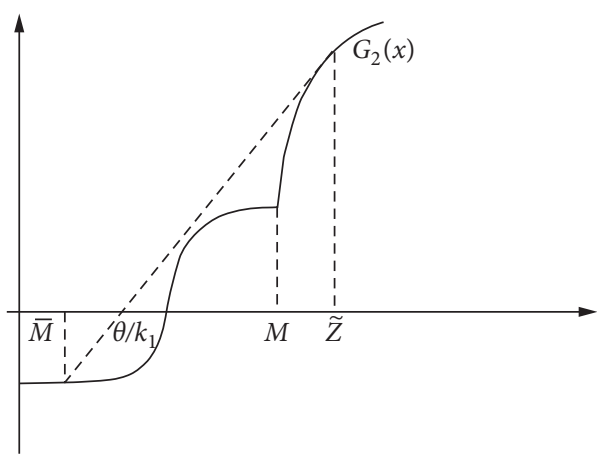

Figure 3: The root to equation (16).

In particular,

$$
\begin{aligned}
& \mathscr{K}\left(G^{\prime}(M-)\right)>G^{\prime}(M-), \\
& \mathscr{K}\left(G^{\prime}(M+)\right)<G^{\prime}(M+) .
\end{aligned}
$$

Then, there must exist $G^{\prime}(M-)<y_{0}<G^{\prime}(M+)$ such that $\mathscr{K}\left(y_{0}\right)=y_{0}$. Define $\left(k_{2} / \theta\right)<z_{1}<M, z_{2}>M$ by the relation $G_{2}^{\prime}\left(z_{1}\right)=G_{2}^{\prime}\left(z_{2}\right)=y_{0}$, which concludes the existence of $z_{1}, z_{2}$. Additionally, we can conclude that the linear interpolation between $G_{2}\left(z_{1}\right)$ and $G_{2}\left(z_{2}\right)$ has to be higher than $G_{2}(\cdot)$ on $\left(z_{1}, z_{2}\right)$, that is,

$$
G_{2}(x)<G_{2}\left(z_{2}\right)+G_{2}^{\prime}\left(z_{2}\right)\left(x-z_{2}\right), \quad z_{1}<x<z_{2} .
$$

Now, we turn to the uniqueness of $z_{1}, z_{2}$. Assume there exists another pair $\left(\widehat{z}_{1}, \widehat{z}_{2}\right)$ with $\left(\theta / k_{2}\right)<\widehat{z}_{1}<M<\widehat{z}_{2}$ satisfying 
$G_{2}\left(\widehat{z}_{2}\right)-G_{2}\left(\widehat{z}_{1}\right)=G_{2}^{\prime}\left(\widehat{z}_{1}\right)\left(\widehat{z}_{2}-\widehat{z}_{1}\right)=G_{2}^{\prime}\left(\widehat{z}_{2}\right)\left(\widehat{z}_{2}-\widehat{z}_{1}\right)$.

Without loss of generality, we assume that $\widehat{z}_{1}>z_{1}$. From (26), we have $G_{2}\left(\widehat{z}_{1}\right)<G_{2}\left(z_{2}\right)+G_{2}^{\prime}\left(z_{2}\right)\left(\widehat{z}_{1}-z_{2}\right)$. So, for $z_{2} \in\left(\widehat{z}_{1}, \widehat{z}_{2}\right)$,

$G_{2}\left(z_{2}\right)>G_{2}\left(\widehat{z}_{1}\right)+G_{2}^{\prime}\left(z_{2}\right)\left(z_{2}-\widehat{z}_{1}\right)>G_{2}\left(\widehat{z}_{1}\right)+G_{2}^{\prime}\left(\widehat{z}_{1}\right)\left(z_{2}-\widehat{z}_{1}\right)$.

However, the linear interpolation between $G_{2}\left(\widehat{z}_{1}\right)$ and $G_{2}\left(\widehat{z}_{2}\right)$ has to be higher than $G_{2}(\cdot)$ on $\left(\widehat{z}_{1}, \widehat{z}_{2}\right)$, that is,

$$
G_{2}\left(z_{2}\right)<G_{2}\left(\widehat{z}_{1}\right)+G_{2}^{\prime}\left(\widehat{z}_{1}\right)\left(z_{2}-\widehat{z}_{1}\right),
$$

which leads to a contradiction.

Proposition 1. Assume $x_{0} \geq \bar{M} e^{-r T}-H(0)$. Let $z, \bar{z}, \tilde{z}$ and $z_{1}, z_{2}$ be determined by (14)-(17), respectively. Then, for the optimization problem (9), the optimal terminal wealth
$X^{\pi^{*}, \beta^{*}}(T)$ is as follows with the multiplier $\beta^{*}>0$ satisfying $E\left[\xi(T) X^{\pi^{*}, \beta^{*}}(T)\right]=x_{0}+H(0)$.

Under the single management fee scheme,

$$
X^{\pi^{*}, \beta^{*}}(T)= \begin{cases}\bar{M}, & \xi(T) \geq \frac{G_{1}^{\prime}(z)}{\beta^{*}}, \\ \frac{1}{k_{1}}\left(\left(\frac{\beta^{*} \xi(T)}{k_{1} \gamma_{2}}\right)^{\left(1 / \gamma_{2}-1\right)}+\theta\right), & \xi(T)<\frac{G_{1}^{\prime}(z)}{\beta^{*}} .\end{cases}
$$

Under the mixed scheme,

Case A. If $k \geq G_{2}^{\prime}(M-)$, then

(I) For $z_{1}<\bar{z}$,

$$
X^{\pi^{*}, \beta^{*}}(T)= \begin{cases}\bar{M}, & \xi(T) \geq \frac{G_{2}^{\prime}(\widetilde{z})}{\beta^{*}}, \\ \frac{1}{k_{2}+k_{3}}\left(\left(\frac{\beta^{*} \xi(T)}{\gamma_{2}\left(k_{2}+k_{3}\right)}\right)^{\left(1 / \gamma_{2}-1\right)}+k_{3} M+\theta\right), & \xi(T)<\frac{G_{2}^{\prime}(\widetilde{z})}{\beta^{*}} .\end{cases}
$$

(II) For $z_{1} \geq \bar{z}$,

$$
X^{\pi^{*}, \beta^{*}}(T)= \begin{cases}\bar{M}, & \xi(T) \geq \frac{G_{2}^{\prime}(\bar{z})}{\beta^{*}}, \\ \frac{1}{k_{2}}\left(\theta+\left(\frac{\beta^{*} \xi(T)}{k_{2} \gamma_{2}}\right)^{\left(1 / \gamma_{2}-1\right)}\right), & \frac{G_{2}^{\prime}\left(z_{1}\right)}{\beta^{*}} \leq \xi(T)<\frac{G_{2}^{\prime}(\bar{z})}{\beta^{*}}, \\ \frac{1}{k_{2}+k_{3}}\left(\left(\frac{\beta^{*} \xi(T)}{\gamma_{2}\left(k_{2}+k_{3}\right)}\right)^{\left(1 / \gamma_{2}-1\right)}+k_{3} M+\theta\right), & \xi(T)<\frac{G_{2}^{\prime}\left(z_{1}\right)}{\beta^{*}}\end{cases}
$$

Case B. If $k<G_{2}^{\prime}(M-)$, then $X^{\pi^{*}, \beta^{*}}(T)$ is given by (31).

Proof. To solve problem (12), we define the Lagrangian of problem (12) as follows:

$$
\mathscr{L}_{i}(X(T), \beta)=E\left[G_{i}(X(T))-\beta \xi(T) X(T)\right],
$$

where $\beta>0$ is a Lagrange multiplier. Note that $G_{i}(\cdot)$ is not concave, so we first derive the concave envelope of $G_{i}(\cdot)$.

Under the single management fee scheme, we let $z$ be the tangent point of the line starting at $\left(\bar{M}, G_{1}(\bar{M})\right)$ to the curve $G_{1}(x), x>\left(\theta / k_{1}\right)$. Then, the concave envelope of $G_{1}$ is given by (see Figure 4 ) 


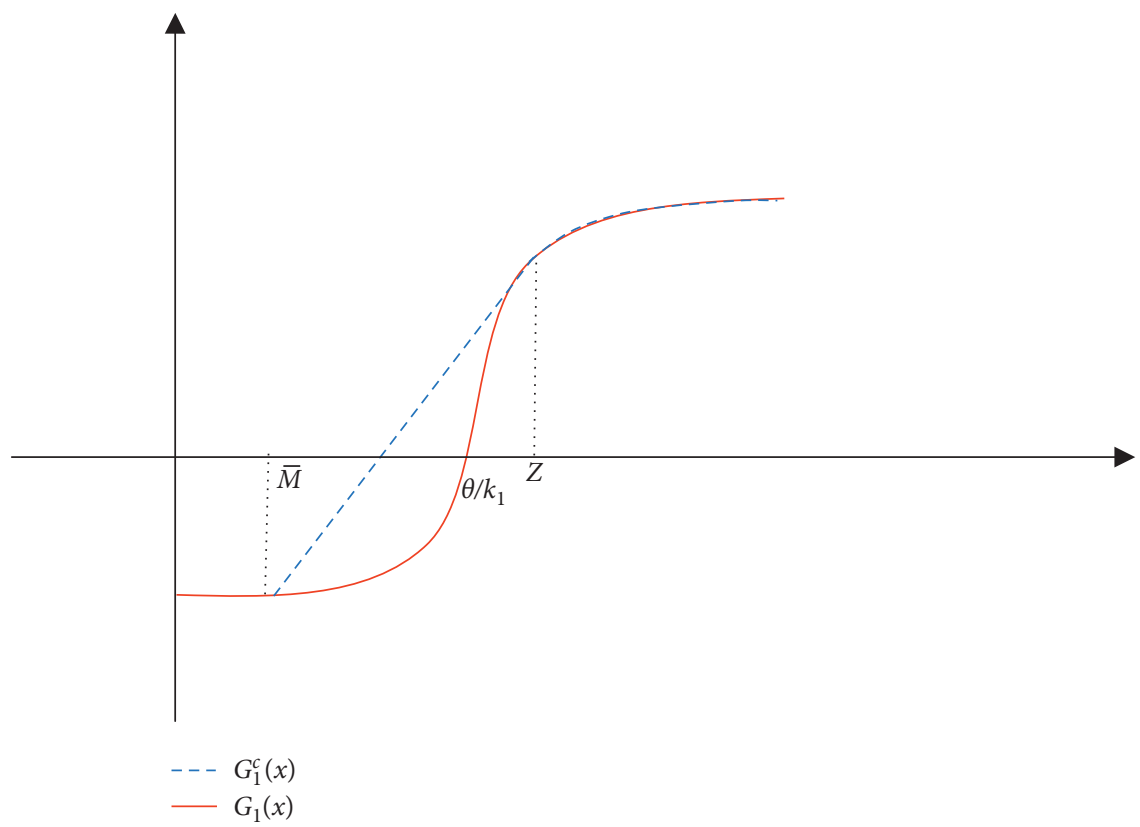

Figure 4: Concave envelope of $G_{1}$.

$$
G_{1}^{c}(x)= \begin{cases}G_{1}(\bar{M})+G_{1}^{\prime}(z)(x-\bar{M}), & \bar{M} \leq x<z, \\ \left(k_{1} x-\theta\right)^{\gamma_{2}}, & x \geq z .\end{cases}
$$

It is easy to verify that for $y>0$,

$$
x^{*}(y) \hat{=} \arg \max _{x \geq \bar{M}}\left\{G_{1}^{c}(x)-x y\right\}= \begin{cases}\frac{1}{k_{1}}\left(\left(\frac{y}{k_{1} \gamma_{2}}\right)^{\left(1 / \gamma_{2}-1\right)}+\theta\right), & 0<y<G_{1}^{\prime}(z), \\ \{\bar{M}, z\}, & y=G_{1}^{\prime}(z), \\ \bar{M}, & y>G_{1}^{\prime}(z) .\end{cases}
$$

Under the mixed fee scheme, Lemma 2 states that there exists a common tangent line with tangent point pair $\left(z_{1}, z_{2}\right)$ to the curve $G_{2}(\cdot),\left(\theta / k_{2}\right)<x<M$ and $G_{2}(\cdot), x>M$.

Case $A$. For $k \geq G_{2}(M-)$, we let $\bar{z}$ be the tangent point of the line starting at $\left(\bar{M}, G_{2}(\bar{M})\right)$ to the curve $G_{2}(x),\left(\theta / k_{2}\right)<x<M$.

(I) For $z_{1}<\bar{z}$, it can be verified that there exists a unique tangent point $\tilde{z}>M$ of the line starting at
$\left(\bar{M}, G_{2}(\bar{M})\right)$ to the curve $G_{2}(x), x>M$. Then, the concave envelope of $G_{2}$ is given by (see Figure 5)

Similar to deriving (35), we can obtain that

$$
x^{*}(y) \widehat{=} \arg \max _{x \geq \bar{M}}\left\{G_{2}^{c}(x)-x y\right\}= \begin{cases}\frac{1}{k_{2}+k_{3}}\left(\left(\frac{y}{\gamma_{2}\left(k_{2}+k_{3}\right)}\right)^{\left(1 / \gamma_{2}\right)}+k_{3} M+\theta\right), & 0<y<G_{1}^{\prime}(z), \\ \{\bar{M}, \tilde{z}\}, & y=G_{2}^{\prime}(\widetilde{z}), \\ \bar{M}, & y>G_{2}^{\prime}(\widetilde{z}) .\end{cases}
$$




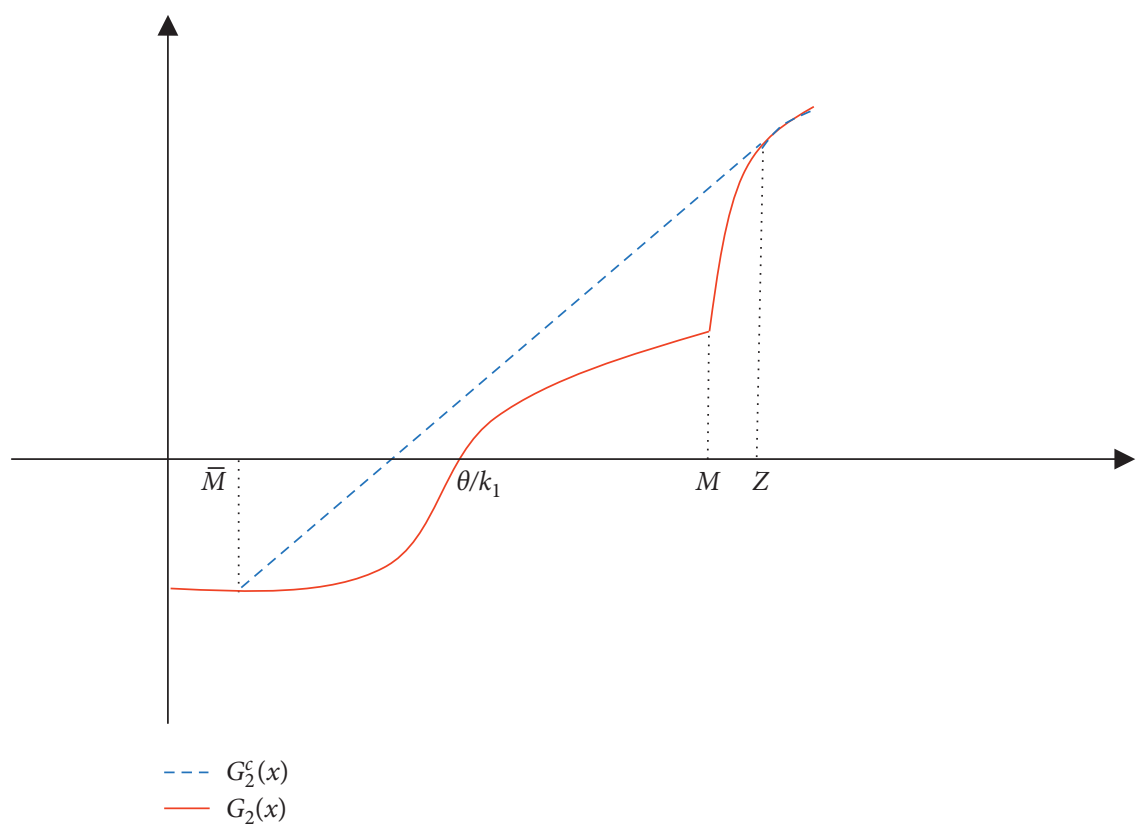

FIGURE 5: Concave envelope of $G_{2}$ for $k \geq G_{2}(M-), z_{1}<\bar{z}$.

(II) For $z_{1} \geq \bar{z}$, the concave envelope of $G_{2}$ is given by (see Figure 6)

So, we have

$$
G_{2}^{c}(x)= \begin{cases}G_{2}(\bar{M})+G_{2}^{\prime}(\bar{z})(x-\bar{M}), & \bar{M} \leq x<\bar{z}, \\ G_{2}(x), & \bar{z} \leq x<z_{1}, \\ G_{2}\left(z_{1}\right)+G_{2}^{\prime}\left(z_{1}\right)\left(x-z_{1}\right), & z_{1} \leq x<z_{2}, \\ G_{2}(x), & x \geq z_{2} .\end{cases}
$$

$$
x^{*}(y)= \begin{cases}\frac{1}{k_{2}+k_{3}}\left(\left(\frac{y}{\gamma_{2}\left(k_{2}+k_{3}\right)}\right)^{\left(1 / \gamma_{2}\right)}+k_{3} M+\theta\right), & 0<y<G_{2}^{\prime}\left(z_{2}\right), \\ \left\{z_{1}, z_{2}\right\}, & y=G_{2}^{\prime}\left(z_{2}\right), \\ \frac{1}{k_{2}}\left(\theta+\left(\frac{y}{k_{2} \gamma_{2}}\right)^{\left(1 / \gamma_{2}-1\right)}\right), & G_{2}^{\prime}\left(z_{2}\right)<y<G_{2}^{\prime}(\bar{z}), \\ \left\{\bar{M}, z_{1}\right\}, & y=G_{2}^{\prime}(\bar{z}), \\ \bar{M}, x y\} & y>G_{2}^{\prime}(\bar{z}) .\end{cases}
$$




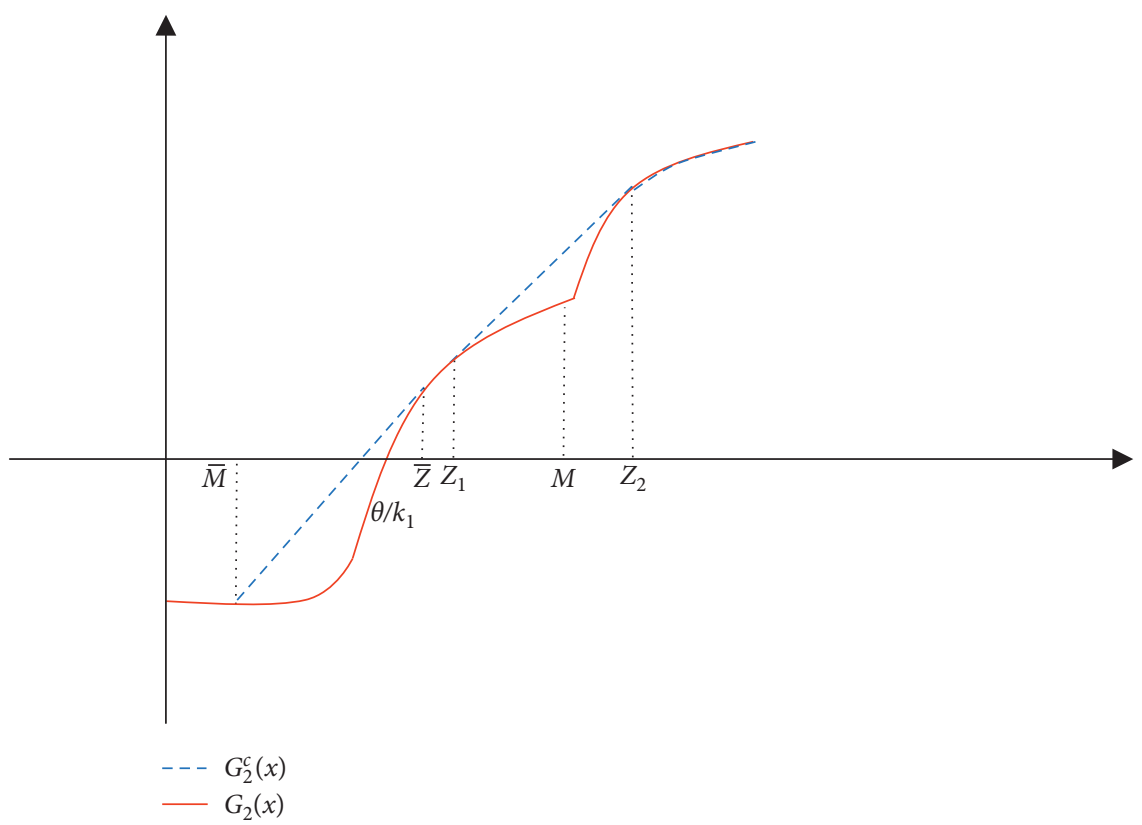

Figure 6: Concave envelope of $G_{2}$ for $k \geq G_{2}(M-), z_{1} \geq \bar{z}$.

Case B. For $k<G_{2}(M-)$, we let $\tilde{z}$ be the tangent point of the line starting at $\left(\bar{M}, G_{2}(\bar{M})\right)$ to the curve $G_{2}(x),\left(\theta / k_{2}\right)<x<M$ (see Figure 7$)$. Then, the concave envelope of $G_{2}$ and $x^{*}(y)$ is given by (36) and (37), respectively.

According to the standard approach to portfolio selection problems, the original problem (12) is equivalent to the following problem:

$$
\begin{cases}\inf _{\beta>0} \max _{X(T)} & \mathscr{L}_{i}(X(T), \beta) \\ \text { s.t. } & X(T) \geq \bar{M}, \text { a.s... }\end{cases}
$$

To solve the above problem, we first consider the following problem:

$$
\begin{cases}\max _{X(T)} & \mathscr{L}_{i}(X(T), \beta) \\ \text { s.t. } & X(T) \geq \bar{M}, \text { a.s } \ldots\end{cases}
$$

It can be verified that for each $\beta>0$, an optimal solution to $(41)$ is

$$
X^{\pi^{*}, \beta}(T)=x^{*}(\beta \xi(T))
$$

Secondly, it remains to solve an optimization problem w.r.t $\beta$ :

$$
\inf _{\beta>0} \mathscr{L}_{i}\left(X^{\pi^{*}, \beta}(T), \beta\right)
$$

By the complementary slackness condition, the optimal solution $\beta^{*}>0$ to problem (43) can be directly obtained from the following equation:

$$
E\left[\xi(T) X^{\pi^{*}, \beta^{*}}(T)\right]=x_{0}+H(0),
$$

which yields the optimal solution $X^{\pi^{*}}, \beta^{*}(T)$ to problem (12).

It is easy to see that in each case, $V(\beta)=E\left[\xi(T) X^{\pi^{*}, \beta}(T)\right]$ is continuous and strictly decreasing in $\beta$. Furthermore, $\lim _{\beta \longrightarrow 0^{+}} V(\beta)=\infty$ and $\lim _{\beta \longrightarrow \infty} V(\beta)=\bar{M} e^{-r T}$. Thus, there exists a unique $\beta^{*}$ satisfying condition (44), which implies that $X^{\pi^{*}, \beta^{*}}(T)$ is feasible to (12). We next show $X^{\pi^{*}, \beta^{*}}(T)$ is indeed the optimal terminal wealth to problem (12). Let $X(T)$ be an arbitrary terminal wealth satisfying the budget constraint in problem (12). Then, we have

$$
\begin{aligned}
E\left[G_{i}\left(X^{\pi^{*}, \beta^{*}}(T)\right)\right]-E\left[G_{i}(X(T))\right] \geq & E\left[G_{i}\left(X^{\pi^{*}, \beta^{*}}(T)\right)-\beta^{*} \xi(T) X^{\pi^{*}, \beta^{*}}(T)\right]+\beta^{*}\left(x_{0}+H(0)\right) \\
& -\left(E\left[G_{i}(X(T))-\beta^{*} \xi(T) X(T)\right]+\beta^{*}\left(x_{0}+H(0)\right)\right) \geq 0,
\end{aligned}
$$

where the first equality holds since the budget constraint holds with equality for $X^{\pi^{*}}, \beta^{*}(T)$ while with inequality for $X(T)$ and the second equality follows from the fact that $X^{\pi^{*}, \beta^{*}}(T)$ solves (41) for the multiplier $\beta^{*}$.
Remark 1. Note that if $\bar{M}=0$, then the loss aversion states a risk-seeking preference for the loss states, and therefore the terminal wealth equals to 0 , which implies in the case of no regulation there is a moral hazard problem due to loss 


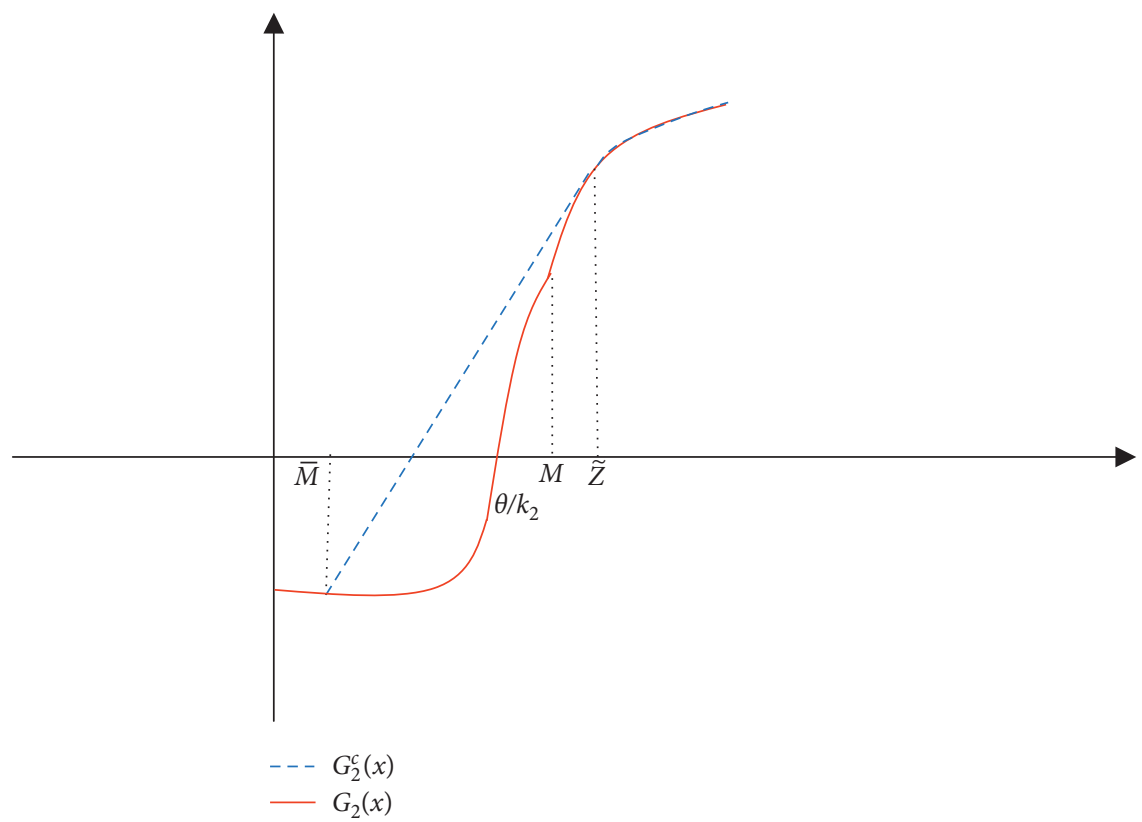

Figure 7: Concave envelope of $G_{2}$ for $k<G_{2}(M-)$.

aversion. In contrast to the unconstrained optimal terminal wealth, the optimal terminal wealth under the PI constraint in bad market scenarios is kept at the level $\bar{M}$ in order to satisfy the PI constraint while the unconstrained terminal wealth is always 0 . Therefore, the PI constraint well protects the DC member's benefits in bad market scenarios.

Remark 2. Different from the derived results under the concave utility in [18], the linear envelope exists both under the single and the mixed fee schemes due to loss aversion. The optimal terminal wealth $X^{\pi^{*}, \beta^{*}}(T)$ is a function of the state price density at maturity $\xi(T)$ and the multiplier $\beta^{*}$. Under the single incentive scheme, the optimal terminal wealth takes a two-region form. When the state price density $\xi(T)$ is relatively low, the optimal terminal wealth $X^{\pi^{*}, \beta^{*}}(T)$ is similar to the smooth utility; when $\xi(T)$ increases above the boundary point of the bad-state region, $\left(G_{1}^{\prime}(z) / \beta^{*}\right)$, the optimal terminal wealth drops to $\bar{M}$ due to the risk-seeking preference in the the domain of losses. Under the mixed incentive scheme, $X^{\pi^{*}, \beta^{*}}(T)$ takes a two-region form for $k<G_{2}^{\prime}(M-)$ and may take a two- or three-region form for $k \geq G_{2}^{\prime}(M-)$ according to the the relative position of the values of $z_{1}$ and $\bar{z}$. In all cases, the optimal terminal wealth is determined by the utility changing points and ends with the minimum guarantee $\bar{M}$ when the state price density at maturity $\xi(T)$ increases above the boundary point of the bad-state region.

After obtaining the optimal terminal wealth, we can derive the closed-form expression for the optimal investment strategy given in the following result.

Proposition 2. Assume that $x_{0} \geq \bar{M} e^{-r T}-H(0)$. Then, for the optimization problem (12), the optimal wealth process and the optimal strategy are given as follows with the multiplier $\beta^{*}>0$ satisfying $E\left[\xi(T) X^{\pi^{*}, \beta^{*}}(T)\right]=x_{0}+H(0)$.

Under the single management fee scheme, the optimal wealth process at time $t$ is given by

$$
X^{\pi^{*}, \beta^{*}}(t)=A_{1}(\xi(t), t)-H(t)
$$

where

$$
\begin{aligned}
A_{1}(\xi(t), t)= & e^{-r(T-t)}\left(\bar{M} \Phi\left(\mathrm{d}_{1, t}\left(G_{1}^{\prime}(z)\right)\right)\right. \\
& +\frac{\theta}{k_{1}}\left(1-\Phi\left(\mathrm{d}_{1, t}\left(G_{1}^{\prime}(z)\right)\right)\right) \\
& +\chi(z) \frac{\Phi^{\prime}\left(\mathrm{d}_{1, t}\left(G_{1}^{\prime}(z)\right)\right)}{\Phi^{\prime}\left(\mathrm{d}_{2, t}\left(G_{1}^{\prime}(z)\right)\right)} \Phi\left(\mathrm{d}_{2, t}\left(G_{1}^{\prime}(z)\right)\right),
\end{aligned}
$$

with $\Phi$ being the standard normal cumulative distribution function and

$$
\begin{aligned}
\mathrm{d}_{1, t}(x) & =\frac{\ln \left(x / \xi(t) \beta^{*}\right)+\left(r-\left(\vartheta^{2} / 2\right)\right)(T-t)}{-\vartheta \sqrt{T-t}}, \\
\mathrm{~d}_{2, t}(x) & =-\mathrm{d}_{1, t}(x)+\frac{\vartheta \sqrt{T-t}}{1-\gamma_{2}} \\
\chi(x) & =\frac{k_{1} x-\theta}{k_{1}}
\end{aligned}
$$

The percentage of wealth invested in the risky assets is given by 


$$
\begin{aligned}
\pi^{*, \beta^{*}}(t)= & \frac{\vartheta}{\sigma\left(1-\gamma_{2}\right)}(\underbrace{X^{\pi^{*}, \beta^{*}}(t)+H(t)}_{\text {total wealth }}-\underbrace{e^{-r(T-t)}\left(\Phi\left(\mathrm{d}_{1, t}\left(G_{1}^{\prime}(z)\right)\right) \bar{M}+\frac{\theta}{k_{1}}\left(1-\Phi\left(\mathrm{d}_{1, t}\left(G_{1}^{\prime}(z)\right)\right)\right)\right)}_{\text {deposit term }} \\
& +\underbrace{\left(1-\gamma_{2}\right) \Phi^{\prime}\left(\mathrm{d}_{1, t}\left(G_{1}^{\prime}(z)\right)\right) \frac{e^{-r(T-t)}}{\vartheta \sqrt{T-t}}(z-\bar{M})}_{\text {risk seeking term }}) .
\end{aligned}
$$

Under the mixed scheme,

Case A. If $k \geq G_{2}^{\prime}(M-)$, then

(I) For $z_{1}<\bar{z}$, the optimal wealth process at time $t$ is given by

$$
X^{\pi^{*}, \beta^{*}}(t)=\bar{A}_{1}(\xi(t), t)-H(t)
$$

$$
\begin{aligned}
\bar{A}_{1}(\xi(t), t)= & e^{-r(T-t)}\left(\bar{M} \Phi\left(\mathrm{d}_{1, t}\left(G_{2}^{\prime}(\widetilde{z})\right)\right)+\frac{k_{3} M+\theta}{k_{2}+k_{3}}\left(1-\Phi\left(\mathrm{d}_{1, t}\left(G_{2}^{\prime}(\widetilde{z})\right)\right)\right)\right. \\
& +\eta(\widetilde{z}) \frac{\Phi^{\prime}\left(\mathrm{d}_{1, t}\left(G_{2}^{\prime}(\widetilde{z})\right)\right)}{\Phi^{\prime}\left(\mathrm{d}_{2, t}\left(G_{2}^{\prime}(\widetilde{z})\right)\right)} \Phi\left(\mathrm{d}_{2, t}\left(G_{2}^{\prime}(\widetilde{z})\right)\right),
\end{aligned}
$$

with

$$
\eta(x)=\frac{\left(k_{2}+k_{3}\right) x-k_{3} M-\theta}{k_{2}+k_{3}} .
$$

The percentage of wealth invested in the risky assets is given by

$$
\begin{aligned}
\pi^{*, \beta^{*}}(t)= & \frac{\vartheta}{\sigma\left(1-\gamma_{2}\right)}(\underbrace{X^{\pi^{*}, \beta^{*}}(t)+H(t)}_{\text {total wealth }} \\
& -\underbrace{e^{-r(T-t)}\left(\Phi\left(\mathrm{d}_{1, t}\left(G_{2}^{\prime}(\widetilde{z})\right)\right) \bar{M}+\frac{k_{3} M+\theta}{k_{2}+k_{3}}\left(1-\Phi\left(\mathrm{d}_{1, t}\left(G_{2}^{\prime}(\widetilde{z})\right)\right)\right)\right)}_{\text {deposit term }} \\
& +\underbrace{\left(1-\gamma_{2}\right) \Phi \prime\left(\mathrm{d}_{1, t}\left(G_{2}^{\prime}(\widetilde{z})\right)\right) \frac{e^{-r(T-t)}}{9 \sqrt{T-t}}(\widetilde{z}-\bar{M})}_{\text {risk-seeking term }} .
\end{aligned}
$$

(II) For $z_{1} \geq \bar{z}$, the optimal wealth process at time $t$ is given by

$X^{\pi^{*}, \beta^{*}}(t)=\bar{A}_{2}(\xi(t), t)-H(t)$, 
where

$$
\begin{aligned}
& \bar{A}_{2}(\xi(t), t)=e^{-r(T-t)}\left(\left(\bar{M}-\frac{\theta}{k_{2}}\right) \Phi\left(\mathrm{d}_{1, t}\left(G_{2}^{\prime}(\bar{z})\right)\right)+\frac{\theta}{k_{2}} \Phi\left(\mathrm{d}_{1, t}\left(G_{2}^{\prime}\left(z_{1}\right)\right)\right)+\frac{k_{2} \bar{z}-\theta}{k_{2}} \frac{\Phi \prime\left(\mathrm{d}_{1, t}\left(G_{2}^{\prime}(\bar{z})\right)\right)}{\Phi \prime\left(\mathrm{d}_{2, t}\left(G_{2}^{\prime}(\bar{z})\right)\right)} \Phi\left(\mathrm{d}_{2, t}\left(G_{2}^{\prime}(\bar{z})\right)\right)\right. \\
& \left.+ \text { left } \psi\left(z_{1}\right) \frac{\Phi \prime\left(\mathrm{d}_{1, t}\left(G_{2}^{\prime}\left(z_{1}\right)\right)\right)}{\Phi \prime\left(\mathrm{d}_{2, t}\left(G_{2}^{\prime}\left(z_{1}\right)\right)\right)} \Phi\left(\mathrm{d}_{2, t}\left(G_{2}^{\prime}\left(z_{1}\right)\right)\right)\right)
\end{aligned}
$$

with

$$
\psi(x)=\left(\left(\frac{k_{2}+k_{3}}{k_{2}}\right)^{\left(\gamma_{2} / 1-\gamma_{2}\right)}\right) \frac{k_{2} x-\theta}{k_{2}} .
$$

The percentage of wealth invested in the risky assets is given by

$$
\begin{aligned}
\pi^{*, \beta^{*}}(t)= & \frac{\vartheta}{\sigma\left(1-\gamma_{2}\right)}(\underbrace{X^{\pi^{*}, \beta^{*}}(t)+H(t)}_{\text {total wealth }} \\
& -\underbrace{e^{-r(T-t)}\left(\frac{k_{2} \bar{M}-\theta}{k_{2}} \Phi\left(\mathrm{d}_{1, t}\left(G_{2}^{\prime}(\bar{z})\right)\right)+\frac{\theta}{k_{2}} \Phi\left(\mathrm{d}_{1, t}\left(G_{2}^{\prime}\left(z_{1}\right)\right)\right)\right)}_{\text {deposit term }} \\
& +\underbrace{\left(1-\gamma_{2}\right)\left(\frac{\bar{z}-\bar{M}}{\vartheta \sqrt{T-t}} \Phi \prime\left(\mathrm{d}_{1, t}\left(G_{2}^{\prime}(\bar{z})\right)\right)+\frac{k_{2} \psi\left(z_{1}\right)-\theta}{k_{2} \vartheta \sqrt{T-t}} \Phi \prime\left(\mathrm{d}_{1, t}\left(G_{2}^{\prime}\left(z_{1}\right)\right)\right)\right)} .
\end{aligned}
$$

Case B. If $k<G_{2}^{\prime}(M-)$, then the optimal wealth process at time $t$ and the percentage of wealth invested in the risky assets are given by (50) and (53), respectively.

Proof. The price of $X^{\pi^{*}, \beta^{*}}(T)$ at $t$ is calculated as follows:

$$
X^{\pi^{*}, \beta^{*}}(t)=\frac{1}{\xi(t)} E\left[\xi(T) X^{\pi^{*}, \beta^{*}}(T) \mid \mathscr{F}_{t}\right]-H(t) .
$$

Note that $\log (\xi(T))$ is a normal distribution. By substituting the expressions for $X^{\pi^{*}, \beta^{*}}(T)$ given in Proposition 1 into (58), we can easily obtain the formula for $X^{\pi^{*}, \beta^{*}}(t)$ by some straightforward calculations.

Let $X^{\pi^{*}, \beta^{*}}(t)=A(\xi(t), t)$. Then,

$$
\mathrm{d} X^{\pi^{*}, \beta^{*}}(t)=I(t, H(t)) \mathrm{d} t+\frac{\partial A}{\partial \xi}(-\xi(t) \vartheta \mathrm{d} W(t))
$$

for some $I(t, H(t))$, and we are only interested in the diffusion part. Comparing it with (3), we have

$$
\pi^{*, \beta^{*}}(t)=-\frac{\vartheta}{\sigma} \frac{\partial A}{\partial \xi} \xi(t)
$$

Some simple calculations yield the results.

Remark 3. Note that, under the concave utility in [18], a risk-seeking term only exists in the optimal policy under the mixed incentive scheme generated by the incentive of the performance fee. However, with the framework of PT, there exists a risk-seeking term both under the single and the mixed incentive schemes due to loss aversion. Therefore, the potential gambling risk-taking policy exists both under the single and the mixed incentive schemes.

\section{Numerical Analysis}

In this section, we do some numerical calculations to investigate the influence of incentive schemes on the optimal terminal wealth.

For all numerical computations, unless specified otherwise, the benchmark data used are as follows: $r=0.03, \mu=$ $0.07, \sigma=0.2, c=1, B=2.25, \gamma_{1}=0.2, \gamma_{2}=0.3, k_{1}=0.05, k_{2}=$ $0.04, k_{3}=0.1, \theta=10, \bar{r}=0.06, x_{0}=20, \bar{M}=50$, and $T=40$.

Figure 8 presents the optimal terminal value $X^{\pi^{*}, \beta^{*}}(T)$ versus $\xi(T)$ for different $\theta$. It is observed that there exists $H^{*}$, such that for $\xi(T) \geq H^{*}$, the optimal terminal value under the mixed incentive scheme is dominated by $X^{\pi^{*}, \beta^{*}}(T)$ under 


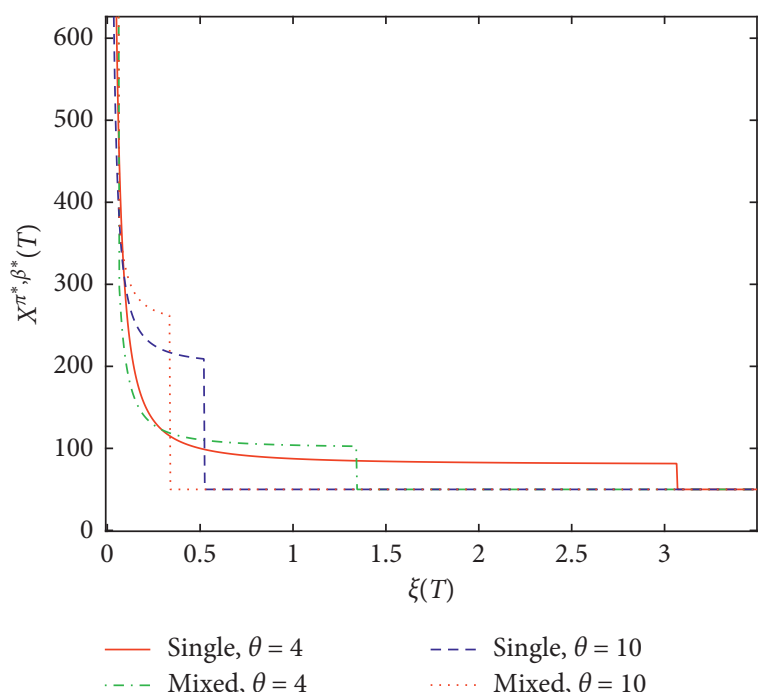

Figure 8: $X^{\pi^{*}, \beta^{*}}(T)$ versus $\xi(T)$ for different $\theta$.

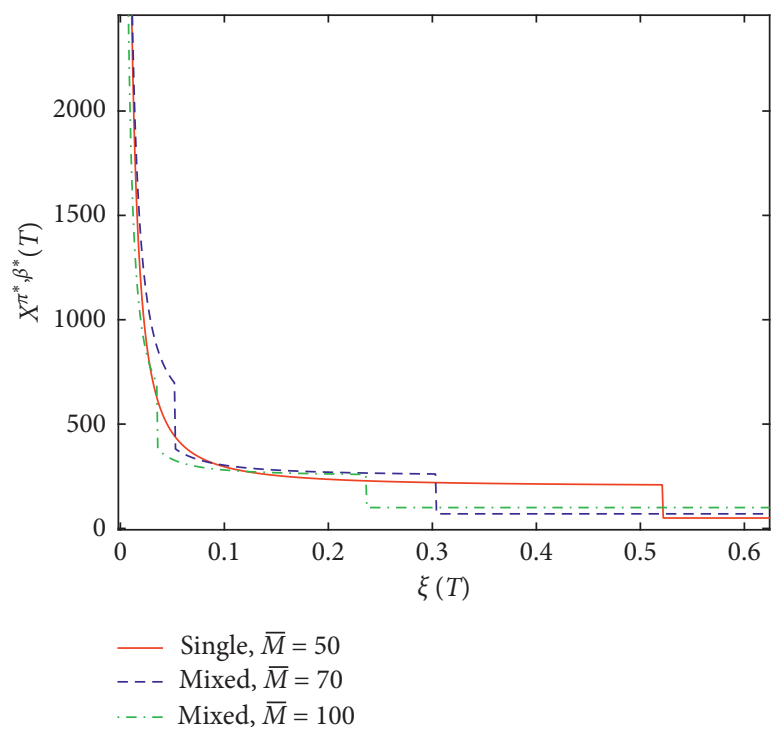

Figure 9: $X^{\pi^{*}, \beta^{*}}(T)$ versus $\xi(T)$ for different $\bar{M}$ under mixed incentive scheme.

the single fee scheme. This is consistent with the intuition that the manger will take more risky strategy to attain the performance fee, which leads to a higher left-tail risk. Furthermore, the mixed incentive scheme also leads to an increase in the optimal terminal wealth of good economic states. We can also note that with the reference point $\theta$ increasing, the badstate region enlarges. This is because the manager is risk seeking towards the terminal wealth. To achieve a higher reference point, the manager invests much more money in the risky asset, which results in a higher left-tail risk.

Figure 9 shows the optimal terminal value $X^{\pi^{*}, \beta^{*}}(T)$ versus $\xi(T)$ under two incentive schemes with different minimum guarantee $\bar{M}$. From it, we can see that a higher left-tail risk under the mixed incentive scheme can be improved by increasing the minimum guarantee. Therefore, we

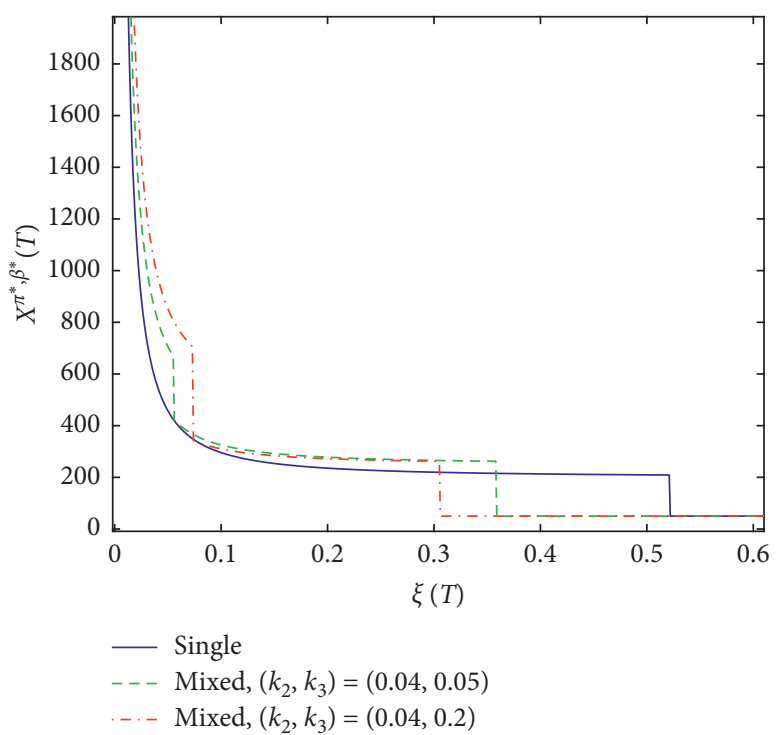

Figure 10: $X^{\pi^{*}, \beta^{*}}(T)$ versus $\xi(T)$ for different $k_{3}$.

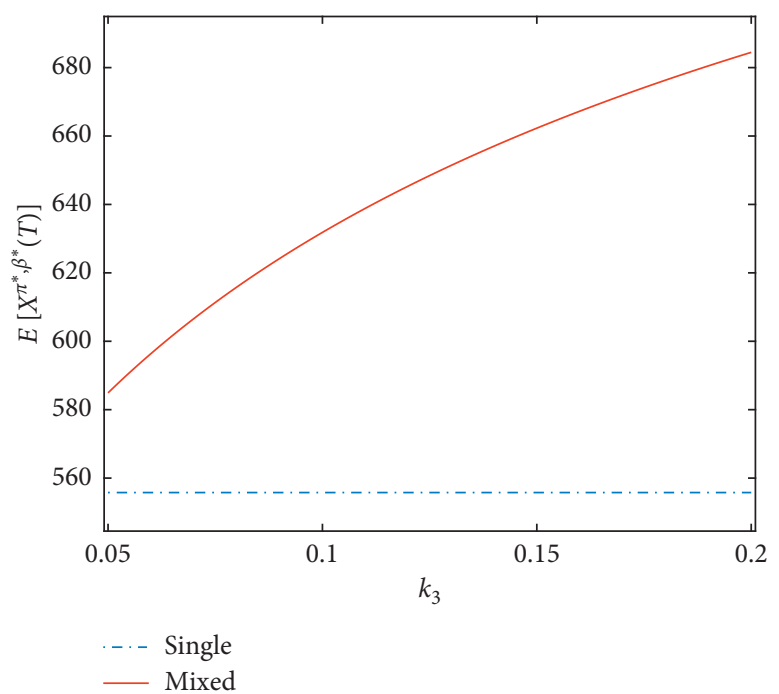

Figure 11: $E\left[X^{\pi^{*}, \beta^{*}}(T)\right]$ versus $k_{3}$.

can control the left-tail risk triggered by the mixed incentive scheme via a stricter PI constraint.

Figure 10 represents the optimal terminal value $X^{\pi^{*}, \beta^{*}}(T)$ versus $\xi(T)$ for different $k_{3}$. We can see that a higher performance fee rate $k_{3}$ leads to enlargement of the bad-state region and increase in the optimal terminal wealth in the good-state region. This is due to the fact that in order to attain the performance fee, the manager takes more risky strategies such that $X^{\pi^{*, \varepsilon}}(T)$ becomes more volatile.

Figure 11 plots the expectation of the optimal terminal value $E\left[X^{\pi^{*}, \beta^{*}}(T)\right]$ versus $k_{3}$. We can see that the expectation of the optimal terminal value $E\left[X^{\pi^{*}, \beta^{*}}(T)\right]$ under the mixed incentive scheme is greater than that under the single incentive scheme, and $E\left[X^{\pi^{*}, \beta^{*}}(T)\right]$ under the mixed incentive scheme increases with $k_{3}$. As pointed out in Figure 10 , to achieve the performance fee, the manager invests 


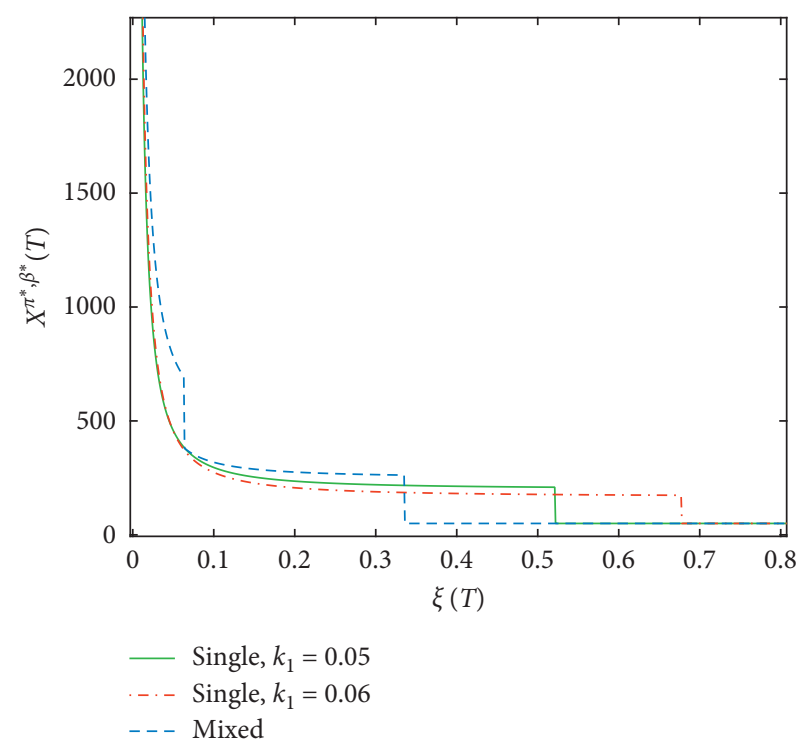

FIgURE 12: $X^{\pi^{*}, \beta^{*}}(T)$ versus $\xi(T)$ for different $k_{1}$.

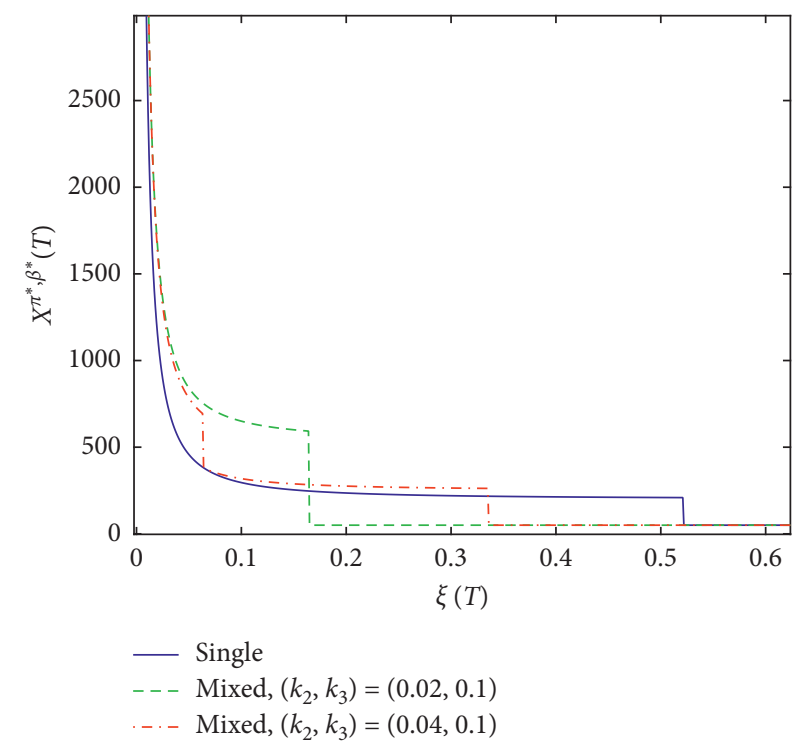

FIgURE 13: $X^{\pi^{*}, \beta^{*}}(T)$ versus $\xi(T)$ for different $k_{2}$.

much more money in the risky asset, which results in a higher expectation.

Figure 12 shows the optimal terminal value $X^{\pi^{*}, \beta^{*}}(T)$ versus $\xi(T)$ for different $k_{1}$. We can see that with $k_{1}$ increasing, the bad-state region shrinks and the optimal terminal wealth in the good-state region decreases. This is because that as $k_{1}$ increases, the manager is paid much more management fee and then the manager takes more prudent strategies such that $X^{\pi^{*} \beta^{*}}(T)$ becomes less volatile. Therefore, a single incentive scheme with a higher management fee rate cannot provide incentive for a manager to increase the optimal terminal wealth.

Figure 13 plots the optimal terminal value $X^{\pi^{*}, \beta^{*}}(T)$ versus $\xi(T)$ for different $k_{2}$. It is seen that a higher fee rate $k_{2}$ leads to shrinkage in the bad-state region shrinks and decrease in the optimal terminal wealth in the good-state region. As explained in Figure 12, with $k_{2}$ increasing, the manager can receive much more management fee and then chooses to take more prudent strategies.

\section{Conclusions}

In this paper, we investigate the optimal portfolio selection problem for a DC plan manager with an S-shaped utility under a single fee scheme and a mixed fee scheme, respectively. We use a concavification technique and a Lagrange dual method to derive closed-form optimal wealth and portfolio processes of the DC pension fund. Due to loss aversion, the potential gambling risk-taking policy exists both under the single and the mixed incentive schemes. In particular, the mixed incentive scheme can induce the manager to take much more risky strategies to achieve a higher optimal terminal value. Financial regulation such as PI constraint can be used to prevent gambling when the performance deteriorates.

\section{Data Availability}

No data were used to support this study.

\section{Conflicts of Interest}

The authors declare that they have no conflicts of interest.

\section{Acknowledgments}

The research was supported by Humanities and Social Science Research Projects in the Ministry of Education (Grant no. 20YJAZH025), the NSF of Jiangsu Province (Grant no. BK20170064), the NNSF of China (Grant no. 11771320), and the QingLan Project of Jiangsu Province.

\section{References}

[1] Y. H. Dong, "Fair valuation of life insurance contract under a correlated jump diffusion model," ASTIN Bull, vol. 41, no. 2, pp. 429-447, 2011.

[2] W. Yu, Y. Yong, G. Guan, Y. Huang, W. Su, and C. Cui, "Valuing guaranteed minimum death benefits by cosine series expansion," Mathematics, vol. 7, no. 9, p. 835, 2019.

[3] Z. M. Zhang, Y. D. Yong, and W. G. Yu, "Valuing equitylinked death benefits in general exponential Lévy models," Journal of Computational and Applied Mathematics, vol. 365, 2020.

[4] Y. Dong, S. Wu, W. Lv, and G. Wang, "Optimal asset allocation for participating contracts under the VaR and PI constraint," Scandinavian Actuarial Journal, vol. 2020, no. 2, pp. 84-109, 2020.

[5] Y. Dong and H. Zheng, "Optimal investment of DC pension plan under short-selling constraints and portfolio insurance," Insurance: Mathematics and Economics, vol. 85, pp. 47-59, 2019.

[6] Y. Dong and H. Zheng, "Optimal investment with S-shaped utility and trading and value at risk constraints: an application to defined contribution pension plan," European Journal of Operational Research, vol. 281, no. 2, pp. 341-356, 2020.

[7] J. Y. Sun, Y. J. Li, and L. Zhang, "Robust portfolio choice for a defined contribution pension plan with stochastic income and 
interest rate," Communications in Statistics-Theory and Methods, vol. 47, pp. 4095-4105, 2018.

[8] W. Yu, P. Guo, Q. Wang et al., "On a periodic capital injection and barrier dividend strategy in the compound Poisson risk model," Mathematics, vol. 8, no. 4, p. 511, 2020.

[9] J.-F. Boulier, S. Huang, and G. Taillard, "Optimal management under stochastic interest rates: the case of a protected defined contribution pension fund," Insurance: Mathematics and Economics, vol. 28, no. 2, pp. 173-189, 2001.

[10] Z. Chen, Z. Li, Y. Zeng, and J. Sun, "Asset allocation under loss aversion and minimum performance constraint in a DC pension plan with inflation risk," Insurance: Mathematics and Economics, vol. 75, pp. 137-150, 2017.

[11] Y. Zeng, D. Li, Z. Chen, and Z. Yang, "Ambiguity aversion and optimal derivative-based pension investment with stochastic income and volatility," Journal of Economic Dynamics and Control, vol. 88, pp. 70-103, 2018.

[12] S. Wang, X. Rong, and H. Zhao, "Optimal investment and benefit payment strategy under loss aversion for target benefit pension plans," Applied Mathematics and Computation, vol. 346, pp. 205-218, 2019.

[13] S. Basak and A. Shapiro, "Value-at-risk-based risk management: optimal policies and asset prices," Review of Financial Studies, vol. 14, no. 2, pp. 371-405, 2001.

[14] S. Basak, A. Pavlova, and A. Shapiro, "Optimal asset allocation and risk shifting in money management," Review of Financial Studies, vol. 20, no. 5, pp. 1583-1621, 2007.

[15] J. N. Carpenter, "Does option compensation increase managerial risk appetite?" The Journal of Finance, vol. 55, no. 5, pp. 2311-2331, 2000.

[16] S. Basak, A. Pavlova, and A. Shapiro, "Offsetting the implicit incentives: benefits of benchmarking in money management," Journal of Banking \& Finance, vol. 32, no. 9, pp. 1883-1893, 2008.

[17] H.-L. Chen and G. G. Pennacchi, "Does prior performance affect a mutual fund's choice of risk? Theory and further empirical evidence," Journal of Financial and Quantitative Analysis, vol. 44, no. 4, pp. 745-775, 2009.

[18] L. He, Z. Liang, Y. Liu, and M. Ma, "Optimal control of DC pension plan management under two incentive schemes," North American Actuarial Journal, vol. 23, no. 1, pp. 120-141, 2019.

[19] D. Kahneman and A. Tversky, "Prospect theory: an analysis of decision under risk," Econometrica, vol. 47, no. 2, pp. 263292, 1979.

[20] A. Tversky and D. Kahneman, "Advances in prospect theory: cumulative representation of uncertainty," Journal of Risk and Uncertainty, vol. 5, no. 4, pp. 297-323, 1992.

[21] G. Guan and Z. Liang, "Optimal management of DC pension plan under loss aversion and value-at-risk constraints," Insurance: Mathematics and Economics, vol. 69, pp. 224-237, 2016. 